\title{
Dissecting strategies to tune the therapeutic potential of SARS-CoV-2- specific monoclonal antibody CR3022
}

Caroline Atyeo, ${ }^{1,2}$ Matthew D. Slein, ${ }^{1}$ Stephanie Fischinger, ${ }^{1,3}$ John Burke, ${ }^{1}$ Alexandra Schäfer, ${ }^{4}$ Sarah R. Leist, ${ }^{4}$ Natalia A. Kuzmina, ${ }^{5,6}$ Chad Mire, ${ }^{5,6}$ Anna Honko, ${ }^{7,8}$ Rebecca Johnson, ${ }^{7,8}$ Nadia Storm,, 78 Matthew Bernett, ${ }^{9}$ Pei Tong, ${ }^{10}$ Teng Zuo, ${ }^{10}$ Junrui Lin, ${ }^{10}$ Adam Zuiani, ${ }^{10}$ Caitlyn Linde, ${ }^{11}$ Todd Suscovich, ${ }^{11}$ Duane R. Wesemann, ${ }^{10}$ Anthony Griffiths,,${ }^{78}$ John R. Desjarlais, ${ }^{9}$ Boris D. Juelg, Jaap Goudsmit, ${ }^{12}$ Alexander Bukreyev, ${ }^{5,6,13}$ Ralph Baric, ${ }^{4,14,15}$ and Galit Alter ${ }^{1}$

'Ragon Institute of MCH, MIT, and Harvard, Cambridge, Massachusetts, USA. ${ }^{2}$ Program in Virology, Division of Medical Sciences, Harvard University, Boston, Massachusetts, USA. ${ }^{3}$ Program in Immunology and Virology, University of Duisburg-Essen, Essen, Germany. ${ }^{4}$ Department of Epidemiology, Gillings School of Global Public Health, University of North Carolina at Chapel Hill, Chapel Hill, North Carolina, USA. ${ }^{5}$ Department of Pathology, University of Texas Medical Branch, Galveston, Texas, USA. ${ }^{6}$ Galveston National Laboratory, Institute for Human Infections and Immunity, University of Texas Medical Branch, Galveston, Texas, USA. Department of Microbiology, Boston University School of Medicine, Boston, Massachusetts, USA. ${ }^{8}$ National Emerging Infectious Diseases Laboratories, Boston University, Boston, Massachusetts, USA. ${ }^{9}$ Xencor, Monrovia, California, USA. ${ }^{10}$ Department of Medicine, Brigham and Women's Hospital; Division of Allergy and Clinical Immunology; and Division of Genetics, Harvard Medical School, Boston, Massachusetts, USA. " 1 SeromYx Systems, Cambridge, Massachusetts, USA. ${ }^{12}$ Departments of Epidemiology and Immunology and Infectious Diseases, Harvard T.H. Chan School of Public Health, Boston, Massachusetts, USA. ${ }^{13}$ Department of Microbiology \& Immunology, University of Texas Medical Branch, Galveston, Texas, USA. ${ }^{14}$ Departments of Microbiology and Immunology and Genetics, School of Medicine, and ${ }^{15}$ Lineberger Comprehensive Cancer Center, University of North Carolina, Chapel Hill, North Carolina, USA.

The rapid spread of severe acute respiratory syndrome coronavirus 2 (SARS-CoV-2), coupled with a lack of therapeutics, has paralyzed the globe. Although significant effort has been invested in identifying antibodies that block infection, the ability of antibodies to target infected cells through Fc interactions may be vital to eliminate the virus. To explore the role of Fc activity in SARS-CoV-2 immunity, the functional potential of a cross-SARS-reactive antibody, CR3022, was assessed. CR3022 was able to broadly drive antibody effector functions, providing critical immune clearance at entry and upon egress. Using selectively engineered Fc variants, no protection was observed after administration of WT IgC1 in mice or hamsters. Conversely, the functionally enhanced Fc variant resulted in increased pathology in both the mouse and hamster models, causing weight loss in mice and enhanced viral replication and weight loss in the more susceptible hamster model, highlighting the pathological functions of Fc-enhancing mutations. These data point to the critical need for strategic Fc engineering for the treatment of SARS-CoV-2 infection.
Conflict of interest: CA is a founder of SeromYx Systems.

Copyright: (c) 2021, Atyeo et al. This is an open access article published under the terms of the Creative Commons Attribution 4.0 International License.

Submitted: August 10, 2020 Accepted: November 25, 2020 Published: January 11, 2021

Reference information: /CI Insight 2021:6(1):e143129.

https://doi.org/10.1172/jci. insight.143129.

\section{Introduction}

The recent pandemic of coronavirus disease 2019 (COVID-19), caused by the severe acute respiratory syndrome coronavirus 2 (SARS-CoV-2), has resulted in millions of infections and more than 1 million deaths globally in a remarkably short period (1). Although most human coronavirus infections cause mild respiratory disease, SARS-CoV and Middle East respiratory syndrome coronavirus (MERS-CoV) resulted in fatality rates of $10 \%$ and $36 \%$, respectively $(2,3)$. Although the precise death counts remain unclear for SARS-CoV-2, fatality rates appear to be lower than those of SARS-CoV and MERS (4). But its alarming rates of spread, linked to transmission during the asymptomatic stage of infection, render this pathogen particularly lethal. Although the development of vaccines against SARS-CoV-2 are underway, therapeutics are urgently needed to support those with more severe infection. Among the therapeutics, several antivirals 
and antiinflammatories are under investigation $(5,6)$. In addition, anti-SARS-CoV-2 monoclonal therapeutics also have been proposed to control and clear the infection.

The coronavirus spike protein (S), found on the surface of coronaviruses, is involved in viral attachment and fusion (7). Treatment with monoclonal antibodies against SARS-CoV S protein has been shown to protect mice from viral pathogenesis (8). Delivery of both neutralizing and non-neutralizing antibodies against the MERS virus afforded protection (9-11), highlighting the potential importance both of blockade of infection and targeted immune-mediated clearance of the virus/virally infected cells in protection from infection. Likewise, both neutralization and antibody-dependent cellular cytotoxicity (ADCC) have been linked to protection in SARS-infected individuals (12) and animal models (13). Given the remarkable infectiousness of SARS-CoV-2, with an estimated $\mathrm{R}_{0}$ approximately 2.5 (14-18), strategies to provide complete protection from infection may require both blocking and postinfection eliminating-antibody functions for maximal immunity. However, data from SARS-CoV-immunized nonhuman primates pointed to the potential role of neutralizing antibodies in enhancing disease, via the induction of inflammatory responses (19), suggesting that caution is warranted in the application of monoclonal antibody therapeutics for SARS-CoV-2 treatment.

To begin to explore the potential immune-protective versus immunopathological role of antibodies, we focused on an antibody derived from a SARS-CoV-infected individual, CR3022, that targets a conserved epitope of the receptor binding domain (RBD) and that binds to the SARS-CoV-2 RBD (20). Because this cross-reactive antibody exhibits limited neutralization, despite binding to conserved determinants on the RBD (21), the antibody offered an opportunity to explore Fc-dependent effects on SARS-CoV-2 not confounded by neutralization. Moreover, given that CR3022 continues to bind to RBD even in the presence of angiotensin-converting enzyme 2 (ACE2) (20-22), CR3022 has the potential to confer eradication of infected cells even in the setting of high ACE2 secretion (23). Thus, here we coupled Fc functional profiling, Fc engineering, and in vivo profiling to examine the role of Fc effector function on the response to SARSCoV-2 infection. Distinct Fc functional profiles resulted in enhancement of disease, pointing to antibody mechanisms of action that may be detrimental when developing antibody therapeutics against the virus.

\section{Results}

CR3022 drives innate immune activity against SARS-CoV-2. Although great effort is underway to identify potent neutralizing antibodies against SARS-CoV-2, it remains uncertain whether neutralization alone, particularly in the upper respiratory tract, will be sufficient to provide complete protection against this highly infectious pathogen. Instead, past studies in MERS infection suggest that additional antibody functions, beyond neutralization, track with protective immunity (13). Thus, the ability to clear virus or infected cells that escape restriction in the upper respiratory tract may be essential to fully prevent disease. Here we focused on a first-in-class monoclonal antibody, initially cloned from a SARS-CoV-infected individual, because of its ability to bind to a mutated neutralization-resistant form of the SARS-CoV S1-RBD (22). CR3022 binds to both SARS-CoV and SARS-CoV-2, with a binding footprint that likely provides the antibody with the ability to bind broadly across SARS-CoV mutants, such as SARS-CoV-2, and to continue to bind in the setting of ACE2 binding to the $\operatorname{RBD}(20,21)$. However, whether this antibody was able to drive additional functions of potential therapeutic utility remained unclear.

To begin, we confirmed the ability of CR3022 to bind to SARS-CoV and SARS-CoV-2 S as well as SARS-CoV-2 RBD and related CoV spike proteins (Figure 1A). As expected, CR3022 bound tightly to the SARS-CoV S, against which it was cloned. The antibody also bound SARS-CoV-2 RBD and S. In contrast, CR3022 did not bind to the MERS S, highlighting the specificity of this antibody for SARS-related viruses. Although it was able to bind to SARS-CoV-2 RBD and potently neutralize SARS-CoV in vitro (22), limited authentic SARS-CoV-2 neutralization was observed even at very high antibody concentrations (Figure 1B) as has been previously observed (21).

Emerging data point to significant differences across monoclonal antibodies in their ability to drive Fc effector functions (24). Both the stoichiometry and geometry of binding have been implicated in modulating antibody effector function (25). Given the peculiar angle with which CR3022 interacts with the SARS-CoV-2 RBD (21), we next examined the ability of CR3022 to drive Fc effector function. CR3022 bound to SARSCoV-2 S was able to bind to $F_{c}$ gamma receptors $2 \mathrm{a}$ and $3 \mathrm{a}$ ( $\mathrm{Fc} \gamma \mathrm{R} 2 \mathrm{a}$ and $\mathrm{Fc} \gamma \mathrm{R} 3 \mathrm{a}$ ) (Figure 1C), whereas no FcR binding was observed with the control EBOV-specific antibody (KZ52) bound to SARS-CoV-2 S, likely due to lack of EBOV monoclonal binding to the SARS-CoV-2 antigen (25). Along the same lines, CR3022 
A

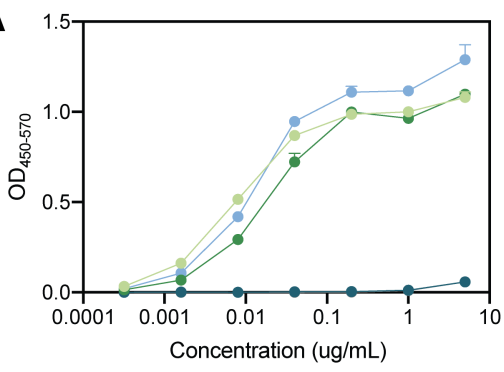

B

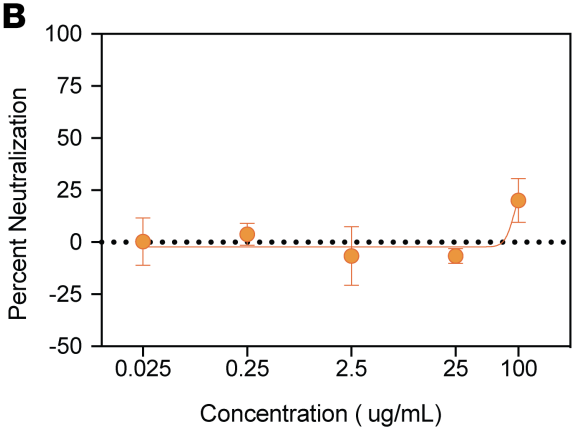

SARS-CoV-2 RBD

- SARS-CoV-2S

- SARS-CoV S

- MERS-CoVS

- EBOVGP

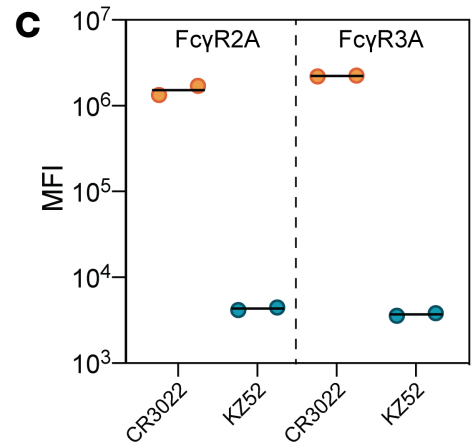

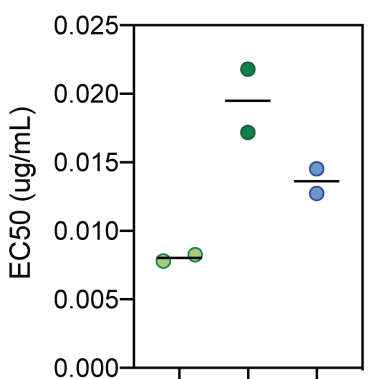

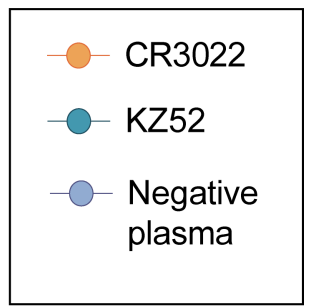

D

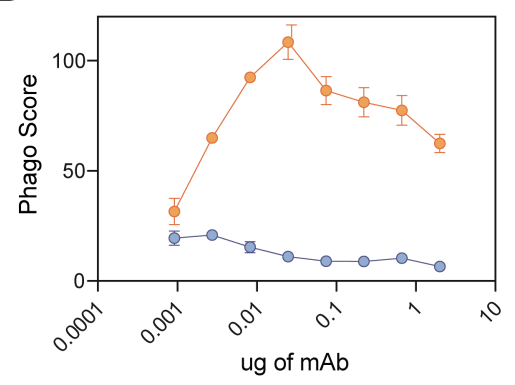

$\mathbf{F}$
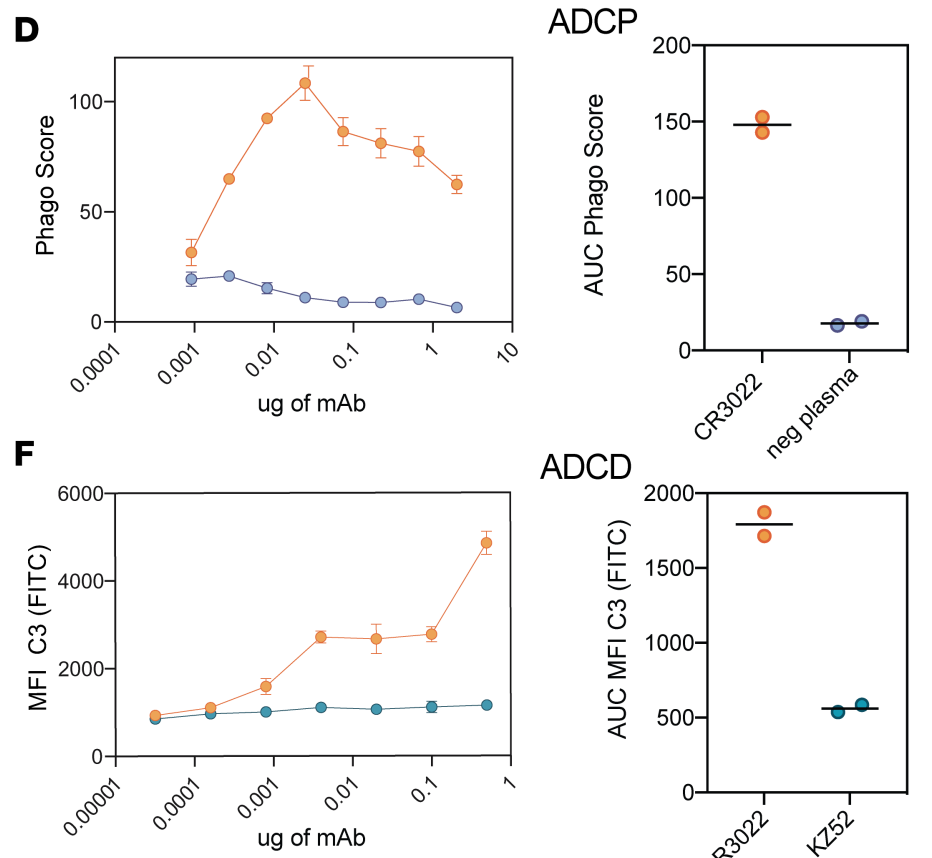

G

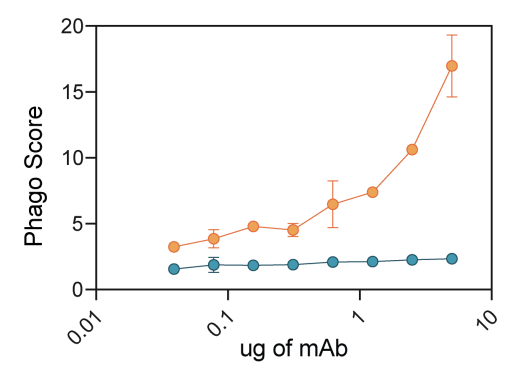

ADCD

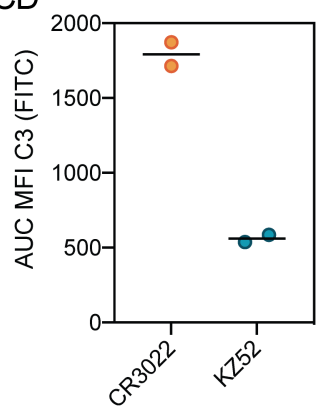

ADNP

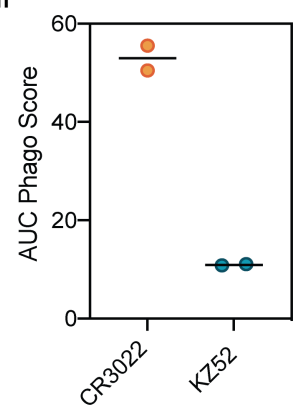

E
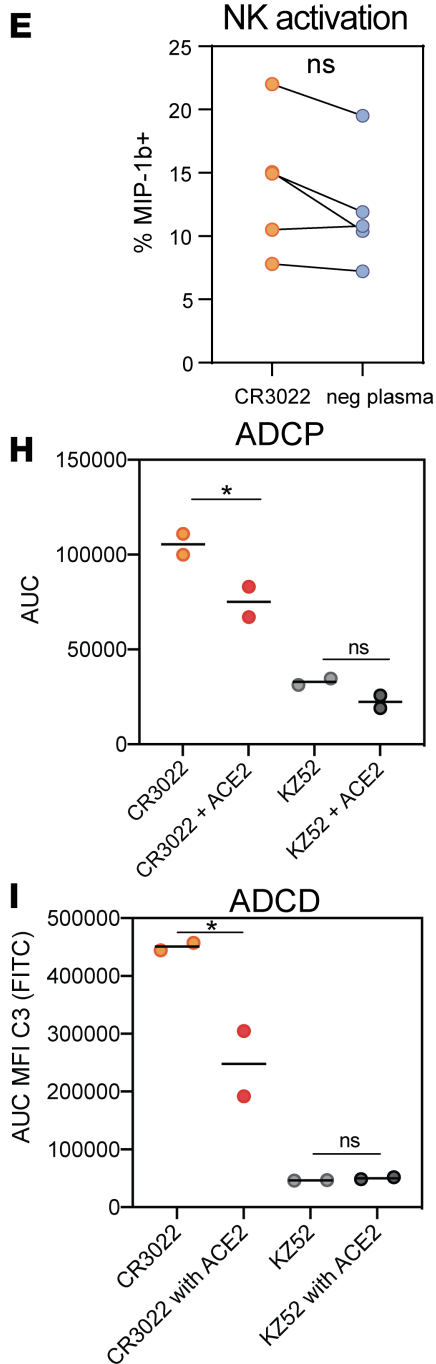
Figure 1. CR3022 drives effector function against SARS-CoV-2. (A) CR3022 was serially diluted and tested for its ability to bind the spike protein of different coronaviruses by ELISA (left). Data are represented as the $\mathrm{OD}_{450}$ values background subtracted from the reference $\mathrm{OD}_{570}$ value. Each dot represents the average of 2 replicates. The bar plot displays the $\mathrm{EC}_{50}$ for each antigen. MERS RBD and Ebola virus (EBOV) glycoprotein are not displayed because their respective $\mathrm{EC}_{50}$ was each infinite. The bars represent the average of 2 replicates, and the error bars represent the standard deviation. (B) CR3022 was serially diluted and preincubated with SARS-CoV-2 before adding to the virus and antibody to Vero E6 cells. Percentage neutralization was determined by the percentage reduction in plaque counts compared with a vehicle control. Data represent the means of triplicates and error bars represent the standard deviation. (C) CR3022 and a control EBOV-specific antibody binding to Fc $\gamma R 2 a$ and $3 a$ were evaluated using Luminex with a serial dilution of CR3022. The AUC was calculated for the MFI values. The bar represents the mean of 2 replicates. (D-C) CR3022 was evaluated for its ability to drive ADCP (D), NK activity (as measured by MIP-1b activity) (E), ADCD (F), and ADNP (C). For the line graphs (left), each dot represents the mean of 2 replicates. For the bar graphs, values are represented as the mean AUC of 8 serial dilutions run in replicate. The error bars for the dots and the AUC represent the standard deviation. For the before-after plot $(\mathbf{E})$, each dot represents the activity of 1 donor after incubation with CR3022 or serum. Significance for NK activation (E) was determined by a Wilcoxon matched pairs signed rank test, ${ }^{*} P<0.05$. ( $\mathbf{H}$ and $\mathbf{~ I ) ~ T h e ~ a b i l i t y ~ o f ~ C R 3 0 2 2 ~ a n d ~ a ~ c o n t r o l ~ E B O V - s p e c i f i c ~ a n t i b o d y ~ ( K Z 5 2 ) ~ t o ~ d r i v e ~ A D C P ~}$ (H) and ADCD (I) in the presence of ACE2 was analyzed. The bars indicate the average AUC of 8 serial dilutions run in 2 replicates. The error bars represent the standard deviation. Significance was determined by a 1-way ANOVA test followed by Tukey's multiple-comparison test. ${ }^{*} P<0.05$.

was able to drive antibody-dependent cellular (monocyte) phagocytosis (ADCP), antibody-dependent NK cell activation (ADNKA, as measured by macrophage inflammatory protein $1 \mathrm{~b}$ [MIP-1b] expression), antibody-dependent complement deposition (ADCD), and antibody-dependent neutrophil phagocytosis (ADNP) (Figure 1, D-G). Moreover, CR3022 was still able to drive antibody effector function, namely ADCP and $\mathrm{ADCD}$, in the presence of ACE2 (Figure 1, H and I). Thus, unlike neutralizing antibodies, many of which compete with ACE2 binding, CR3022 may still drive antibody-effector clearance of virus or infected cells even upon ACE2 upregulation following infection (26).

Comparison of CR3022 and other SARS-CoV-2 monoclonals. To fully probe the therapeutic potential of CR3022, we compared the functional activity of CR3022 with recently published (27) or discovered monoclonal antibodies specifically cloned from SARS-CoV-2-infected individuals. Specifically, the ability of CR3022 to mediate ADCP, NK activation, ADCD, and ADNP was compared with 2 neutralizing antibodies (B38 and 0012C10) and 1 non-neutralizing antibody (0012E4) (Table 1). In addition, we used an EBOV-targeting antibody (KZ52) as a negative control. All SARS-CoV-2 RBD-targeting antibodies drove similar levels of ADCP and ADNP (Figure 2, A and B), with CR3022 comparable to all other SARSCoV-2 monoclonals. Conversely, CR3022 drove slightly more NK activation, as represented by MIP-1b secretion (Figure 2C), and significantly more complement deposition (ADCD) compared with the other 3 SARS-CoV-2 RBD-targeting antibodies (Figure 2D). Thus, CR3022 exhibited similar if not superior antibody-effector function compared with neutralizing and other non-neutralizing antibodies. Moreover, given the emerging role for $\mathrm{ADCD}$ in providing vaccine-mediated protection in vivo (28), the ability of CR3022 to facilitate ADCD makes CR3022 an ideal candidate for potential therapeutic applications.

$F_{c}$ engineering can tune CR3022 effector function. Although $\mathrm{Fc}_{\mathrm{c}}$ effector functions have been linked to protection from MERS infection in mice (13), data have also emerged pointing to the potentially deleterious role of Fc effector functions in enhancing SARS-CoV disease $(19,29,30)$. Thus, using a simple Fc-engineering approach where the variable domains of CR3022 are swapped onto the $\mathrm{Fc}_{\mathrm{c}}$ domain of distinct $\mathrm{Fc}$ variants with previously defined point mutations known to alter antibody interactions with $\mathrm{Fc}_{\mathrm{c}}$ receptors, we generated a small panel of CR3022 Fc variants, able to selectively augment phagocytosis, NK cell function, and complement activity. Given clear overlap and concordance in human IgG1 performance across human and mouse effector functions (31), we focused on known mutations in human IgG1 and compared human and mouse $\mathrm{Fc}$ receptor binding as well as human antibody effector functions. These mutations in the $\mathrm{CH} 2$ or $\mathrm{CH} 3$ of the $\mathrm{Fc}$ of IgG1 were previously identified to alter binding to $\mathrm{Fc} \gamma \mathrm{Rs}$, resulting in enhancement or reduction of $\mathrm{Fc}$ effector function. Specifically, 4 mutants were explored: a mutant able to enhance all $\mathrm{Fc}$ effector functions (EFTEA, ref. 32), 2 mutations known to enhance ADCC and ADCP (SDIE and SDIESA, ref. 33), and a mutation that knocks out all Fc function (N297Q, ref. 34). To begin characterizing the function of these variants, we analyzed the ability of these mutants to bind both human Fc $\gamma$ Rs and mouse Fc $\gamma$ receptors (mFc $\gamma$ Rs) (Figure 3A). EFTEA showed similar binding to the human Fc $\gamma$ Rs as WT CR3022. SDIE exhibited slightly higher binding to the human Fc $\gamma$ Rs compared with WT, whereas SDIESA had a slight and selective reduction in binding to the human Fc $\gamma$ R3A and Fc $\gamma$ R3B. Conversely, the Fc-knockout mutation, N297Q, resulted in a near-complete loss of Fc receptor binding.

Although the variants bound the mouse Fc $\gamma$ Rs with lower affinity than the human Fc $\gamma$ Rs, most variants retained considerable binding to $\mathrm{mFc} \gamma \mathrm{R} 4$, an activating receptor in mice that has been implicated in antibody effector function (35). In particular, SDIE exhibited the highest binding to the mouse Fc $\gamma$ Rs 
Table 1. Neutralizing ability of antibodies that target the RBD of SARS-CoV-2

\begin{tabular}{cc}
\hline mAb & Neutralization \\
\hline CR3022 & - \\
B38 & + \\
$0012 C 10$ & + \\
$0012 E 4$ & -
\end{tabular}

The table lists the neutralizing capacity of a panel of monoclonal antibodies that bind to the SARS-CoV-2 RBD, with B38, 0012C10, and 0012E4 cloned from infected individuals. B38 and 0012C10 are able to neutralize the virus, whereas CR3022 and 0012E4 have limited to no neutralizing capacity.

compared with all other variants. Consistent with Fc $\gamma \mathrm{R}$ binding profiles, the EFTEA mutant exhibited enhanced pan-functionality, with a selective increase in ADCD (Figure 3E). Conversely, both SDIE and SDIESA exhibited enhanced ADCP and ADNP but not ADCD activity, with SDIE exhibiting enhanced ADNP compared with the other mutants (Figure 3, C-E). Finally, SDIESA exhibited the highest ADCP activity and NK activation, as measured by MIP-1b expression, of the group (Figure 3, B and C), highlighting the distinct functional profiles of each of the modifications. Although some residual binding was noted for N297Q on FcyR2A (Figure 3A), near-complete loss of antibody function was observed with this $\mathrm{Fc}$ variant (Figure 3, B-E), driving effector function at a similar level as the EBOV-specific control antibody, underscoring the dominant silencing effect of this mutation. Overall, these data highlight a range of functional variation across the Fc variants, enabling the down-selection of 3 variants for in vivo analysis: WT, Fc-knockout (Fc-KO), and pan-functional SDIE variant, which exhibited the highest binding to mouse Fc $\gamma$ Rs (Figure 3A).

Dissecting therapeutic $F_{C}$ signals of protection. Using these down-selected $F_{C}$ variants, we next aimed to probe the role of Fc effector function in protection from infection and disease (36). BALB/c mice were treated 12 hours following SARS-CoV-2 infection to probe for therapeutic protective Fc functions. Specifically, BALB/c mice were infected with $10^{5} \mathrm{PFU}$ of mouse-adapted SARS-CoV-2 i.n.; treated with $200 \mu \mathrm{g}$ of the WT, Fc-KO, or control antibody or $100 \mu \mathrm{g}$ of the Fc-enhanced antibody i.p.; and monitored for 2 days for lung viral titer and weight loss, with 5 mice per group (Figure 3, F and G).

Strikingly, slightly higher, but nonsignificant, differences were observed in viral replication using the WT CR3022 IgG1 compared with the control antibody and the WT antibody (Figure 3G). Moreover, reduction in viral load was observed with the Fc-enhanced CR3022 variant (Figure 3G). However, significant weight loss occurred in both WT and Fc-enhanced variant-treated mice and minimal to no weight loss in mice treated with the Fc-KO variants (Figure 3G). Thus, while the WT CR3022 IgG1 and the Fc-enhanced CR3022 variants showed divergent virologic effects, both variants led to enhanced pathology. The disconnect between viral load and weight loss for the Fc-enhanced CR3022 antibody raised the possibility that the Fc-enhanced variant may have pathological consequences.

To further understand the role of Fc-enhanced pathology, we next tested the therapeutic benefit of the CR3022 variants in a more pathological model of SARS-CoV-2 infection $(37,38)$. Syrian golden hamsters are highly susceptible to SARS-CoV-2 infection and develop severe infection after challenge. Thus, using this model, we assessed the panel of Fc-engineered monoclonals. Hamsters were challenged i.n. with $10^{6} \mathrm{PFU} / \mathrm{mL}\left(10^{7} \mathrm{TCID}_{50}\right)$ of SARS-CoV-2, and 1 day after infection, treated with 5 $\mathrm{mg} / \mathrm{kg}$ IgG1, Fc-enhanced, Fc-KO, or a control antibody, with 5 hamsters per group. Weight was monitored daily, and lung viral titers were determined 3 days after infection (Figure 4, A and B). Similar to the results observed in the mouse model (Figure 3G), the WT CR3022 had no impact on viral load compared with control antibody-treated animals (Figure 4A). In contrast to mice, hamsters did not experience any benefit from the Fc-KO antibody (Figure 4A), likely due to the more severe nature of the infection in this model. Conversely, hamsters treated with the Fc-enhanced CR3022 exhibited increased viral load in the lung and increased weight loss (Figure 4, A and B). Despite the viral load disparity across the mice and hamsters, both models exhibited increased weight loss upon treatment with the Fc-enhanced CR3022 (Figure 3G and Figure 4B), suggesting similar host responses to the Fc-enhanced monoclonal. Thus, collectively, these data point to the critical importance of balancing Fc effector function to temper pathology in susceptible populations. 
A

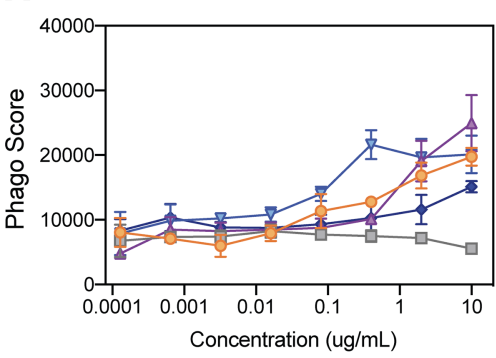

ADCP

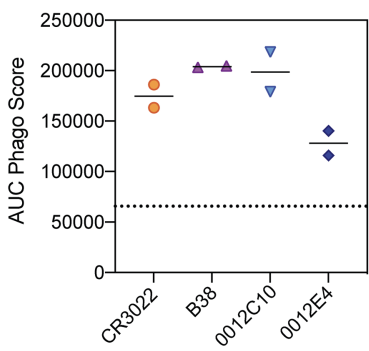

B

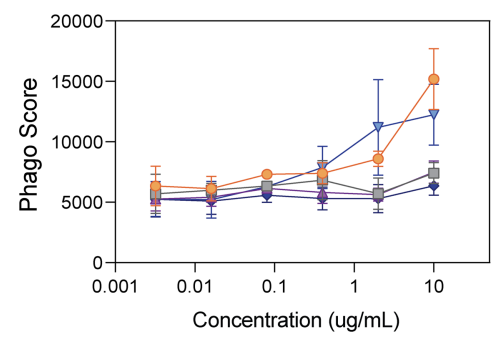

ADNP

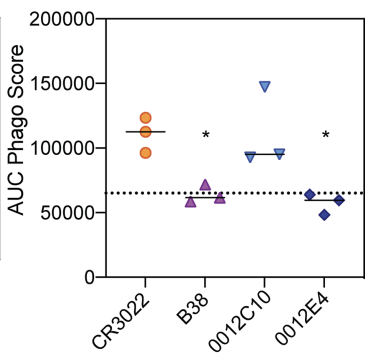

C

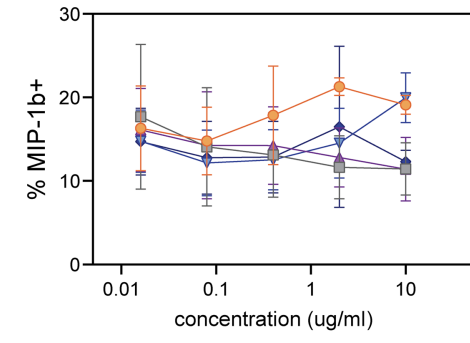

NK activation

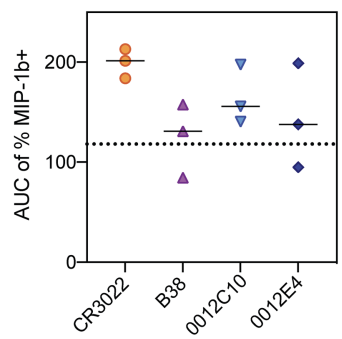

D

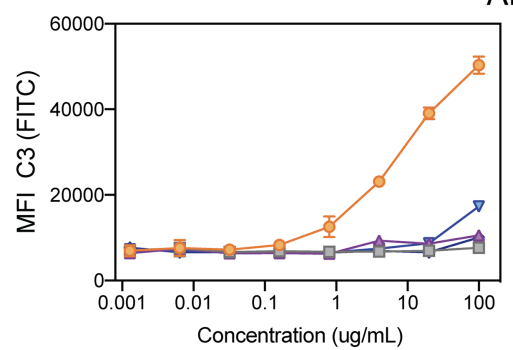

$A D C D$

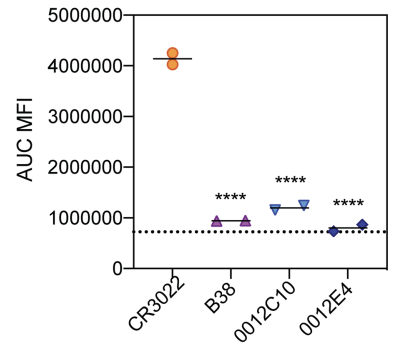

Figure 2. CR3022 possesses comparable Fc activity to neutralizing and non-neutralizing SARS-CoV-2 monoclonal antibodies. (A-D) CR3022 was tested for its ability to drive ADCP (A), ADNP (B), NK cell activation (C), or ADCD (D) compared with other SARS-CoV-2 RBD-targeting monoclonals. For the line graphs, each dot represents the mean of 2 replicates. For NK cell activation, 3 donors were used. For the bar graphs, the values are represented as the AUC of serial dilutions. The dotted line represents the average AUC value for the control EBOV-targeting antibody (KZ52). The error bars represent the standard deviation between the replicates. Significance was determined by an ordinary 1-way ANOVA followed by Tukey's multiple-comparison test. ${ }^{*} P<0.05,{ }^{* * *} P<0.0001$.

\section{Discussion}

Given the rapid spread of SARS-CoV-2, therapeutics are urgently needed to not only prevent but also treat COVID-19. Among the strategies, passive transfer of monoclonal antibodies, which are able to both drive directed antiviral activity and also tune the immune system, represent an ideal class of therapeutics, potentially suited for both prevention and therapy. However, emerging data pointing to the possibility of antibody enhancement of disease following vaccination against SARS-CoV have raised the importance of carefully considering the role of the antibody Fc in SARS-CoV-2 therapeutic design. Here we focused on a first-in-class cross-SARS monoclonal antibody, CR3022, which interacts with a conserved region of the RBD. Although the antibody itself was highly functional, even in the presence of ACE2 (Figure 1), additional Fc engineering was performed, allowing us to gauge the therapeutic benefits of Fc activity (Figure 3). Surprisingly, the Fc-enhanced CR3022 antibody conferred some viral control when administered in mice but was accompanied by significant morbidity in both mice and hamsters, potentially by promoting an inflammatory response.

Past reports have shown some evidence of antibody-dependent enhancement (ADE) of SARS infection. Although many of these studies rely on in vitro systems with high levels of virus, diluted serum, or nonfunctional antibodies $(39,40)$, a more recent study in nonhuman primates pointed to a disease-enhancing role of modified vaccinia Ankara-induced SARS-CoV neutralizing antibodies (19). In vitro data suggested that despite the neutralizing activity of the vaccine-induced antibodies, ADE in SARS infection was caused by Fc $\gamma$ R2-mediated activation of myeloid cells $(41,42)$. ADE is most well documented during Dengue virus infection, in which previous infection places an individual at risk, upon reinfection, to develop Dengue hemorrhagic fever/ Dengue shock syndrome (DHF/DSS). This disease enhancement is thought to occur due to the presence of antibodies directed at a different Dengue serotype. Specifically, subneutralizing levels of preexisting antibodies to one serotype that have limited cross-reactive neutralizing capacity to a second serotype are enriched in children who develop DHF/DSS following exposure to a new serotype of the virus (43). Moreover, in vitro, low levels of neutralizing antibodies have been shown to facilitate viral entry into myeloid cells following Fc $\gamma \mathrm{R}$ engagement, resulting in enhanced infection and consequent inflammation (44). However, it is critical to note that limited evidence exists for SARS-CoV-2 infection of myeloid cells $(45,46)$. Instead, SARS-CoV-2 target 
A

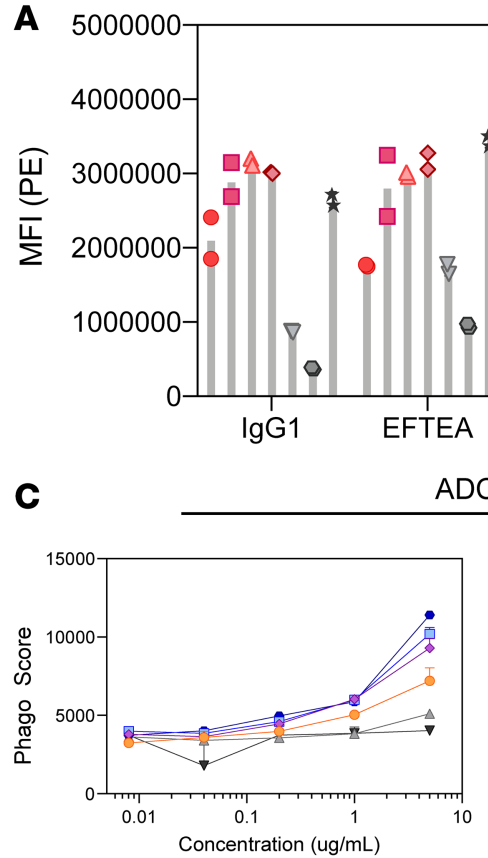

C

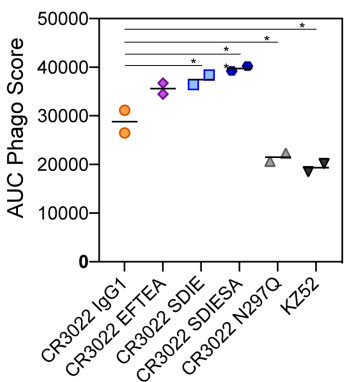

E

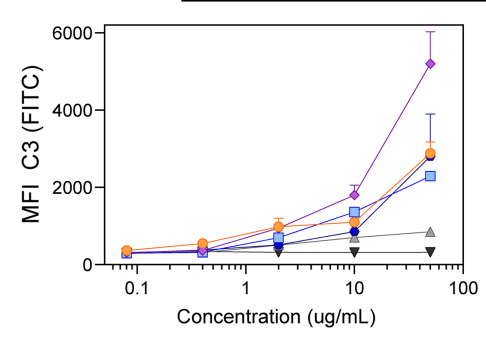

ADCD

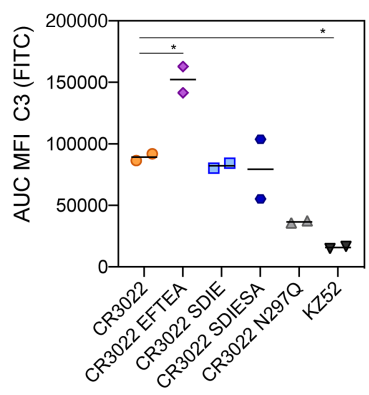

B

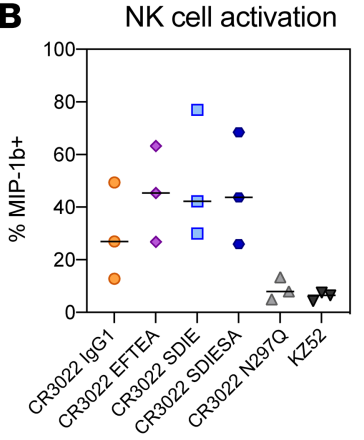

ADNP

D

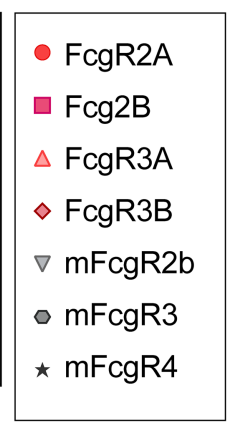

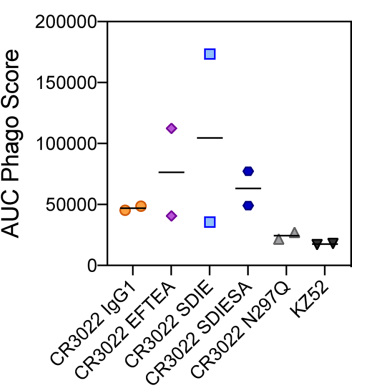

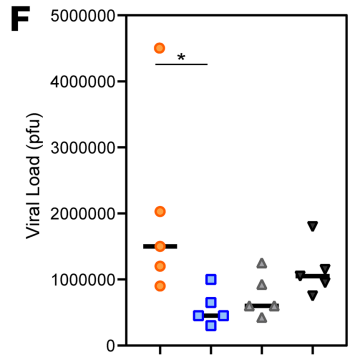

G

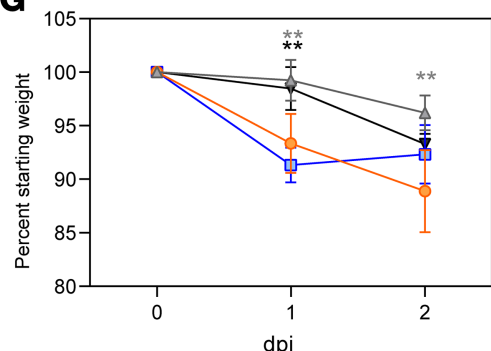

$\neg$ CR3022 IgG1 $\prec$ CR3022 EFTEA $\square-$ CR3022 SDIE

- CR3022 SDIESA $\triangle$ CR3022 Fc-KO $\rightarrow$ Control Antibody

Figure 3. CR3022 can be enhanced through Fc engineering. (A) Each CR3022 variant was analyzed for its ability to bind the human Fc $\gamma R 2 a$, Fc $\gamma R 2 b$, Fc $\gamma R 3 a$, and Fc $\gamma$ R3b or the mouse Fc $\gamma R 2 b, F c \gamma R 3$, and Fc $\gamma R 4$ by Luminex. Each bar represents the average MFI and error bars show standard deviation. (B) The CR3022 variants were analyzed for their ability to drive NK activation, as measured by MIP-1b activity. The bar graphs represent the average of 3 donors, and the error bars represent the standard deviation. For AUC, significance was determined by an ordinary 1-way ANOVA followed by Tukey's multiple-comparison test. ${ }^{*} P<0.05$. (C-E) The CR3022 variants were evaluated for their ADCP (C), ADNP (D), and ADCD (E) activity. The dots on the line graph represent the average of 2 replicates. The bar graphs represent the average AUC of 8 serial dilutions run as replicates, and the error bars represent the standard deviation. For AUC, significance was determined by an ordinary 1-way ANOVA followed by Tukey's multiple-comparison test. ${ }^{*} P<0.05$. ( $\mathbf{F}$ and $\mathbf{G}$ ) BALB/c mice (5 per group) were treated with a CR3022 variant or control therapeutically. Lung viral titers were determined 2 days postinfection (dpi) (F), and weight was monitored daily (G). For viral titer (F), significance was determined by an ordinary 1 -way ANOVA followed by Tukey's multiple-comparison test. ${ }^{*} P<0.05$. For weight (G), significance was determined by an ordinary 1-way ANOVA followed by Tukey's multiple-comparison test. ${ }^{*} P<0.05$, ${ }^{*} P<0.01$, between the respective antibody, indicated by the color of the asterisk, and the CR3022 WT. For weight (G), each dot represents a mouse, and the error bars represent the standard deviation.

cells do not express FcyR, and thus enhanced infection is unlikely to occur in the same manner as for Dengue viral infection. Conversely, SARS-CoV-2 target cells do express the neonatal Fc receptor, FcRn (47, 48). Whether enhancement can be caused by FcRn remains unclear. Yet, enhanced $F c \gamma R$ engagement may deliver virus preferentially to endosomal compartments in phagocytes, resulting in viral sensing and inflammatory responses, that may then lead to inflammation, cellular recruitment, and potential pathology. Thus, antibodies in SARS-CoV-2, unlike Dengue viral infection, may cause enhanced inflammation rather than enhanced infection. Importantly, here both FcyR-engaging antibodies drove pathology in both mice and hamsters but showed differences in viral loads. These data point to a disconnect between viral load and pathogenesis that may be dissected in the future with in-depth immunohistopathological studies across the 2 animal models. 
A

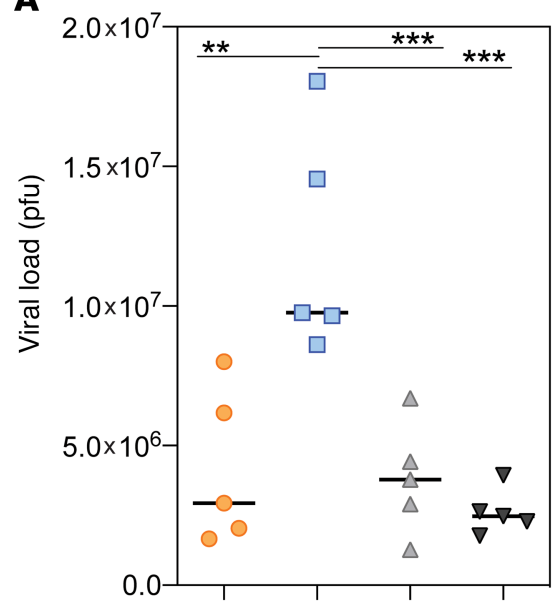

B

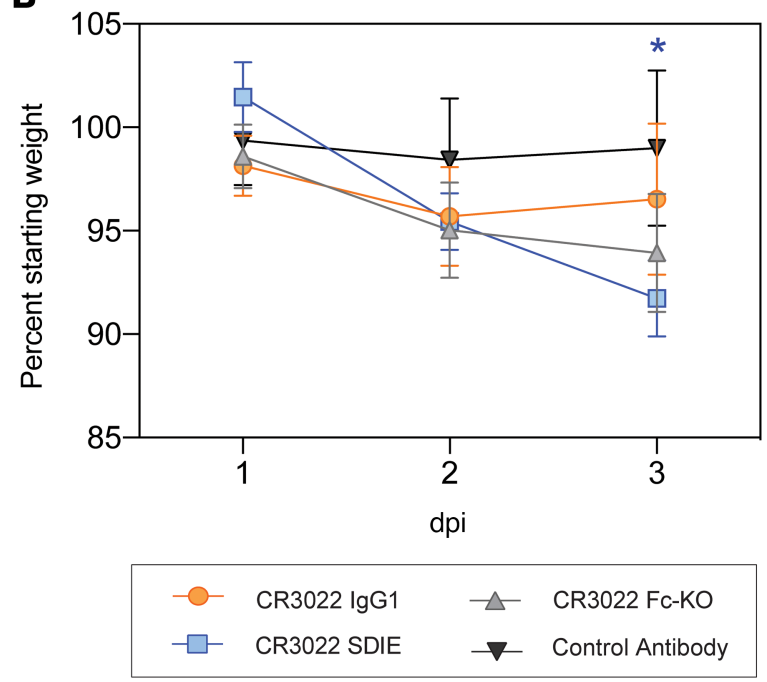

Figure 4. Increased Fc function causes enhancement of disease in vivo. Hamsters ( 5 per group) were challenged with SARS-CoV-2 and treated with a CR3022 variant or control antibody $1 \mathrm{dpi}$. Lung viral titers were determined $3 \mathrm{dpi}(\mathbf{A})$, and weight was monitored daily (B). For viral titers (A), significance was determined by an ordinary 1-way ANOVA followed by Tukey's multiple-comparison test. ${ }^{* *} P<0.01,{ }^{* *} P<0.001$. For weight (B), significance was determined by 2-way ANOVA test followed by Tukey's multiple-comparison test. ${ }^{*} P<0.05$ between the respective antibody and the CR3022 WT. For weight (B), each dot represents the average of 5 hamsters, and the error bars represent the standard deviation.

It is critical to note that antibodies elicited by infection and vaccine platforms or monoclonal antibodies do not have the same Fc binding profiles as those induced by modified vaccinia Ankara (MVA) vaccination that was previously associated with SARS-CoV-enhanced disease in macaques (49). Importantly, distinct Fc binding profiles can be generated following infection and vaccination, driven by altered Fc subclass selection and Fc glycosylation (49). Moreover, previous studies have clearly illustrated striking differences in antibody functional profiles across MVA-, pox virus-, adenovirus-, and protein-based immunization strategies (50). Thus, it is plausible that polyclonal pools of antibodies, with neutralizing properties and balancing Fc receptor binding profiles via balanced Fc glycosylation, may provide protection in the absence of disease. Along these lines, recent vaccine studies point to a positive predictive role of polyclonal $\mathrm{Fc}$ recruiting directed at both the whole spike and RBD of SARS-CoV-2 $(28,51)$. Similarly recent monoclonal therapeutic studies with neutralizing WT IgG1 demonstrate limited evidence of disease in humans (52).

Despite the significant loss of most Fc effector functions in the Fc-KO variant (Figure 3), this variant retained low-level binding to human $\mathrm{Fc} \gamma \mathrm{R} 2 \mathrm{~A}$, involved in phagocytosis in humans (53). This remaining Fc receptor binding may have contributed to low but sufficient levels of immune complex-based activation in the susceptible hamster model, where even the Fc-KO variant drove weight loss. These data point to the ultrasensitive nature of the hamster model. Although mice exhibit more attenuated disease, hamsters suffer highly pathological responses to SARS-CoV-2 (37). Whereas the Fc-KO exhibited reduced viral and no weight loss in the mice, the administration of the Fc-enhanced variant resulted in pathology. Conversely, the more susceptible hamster model showed no benefit with any of the Fc variants and instead exhibited the same enhanced pathology with the Fc-enhanced. Thus, Fc enhancement may represent a liability across the disease spectrum. However, it is critical to note that many distinct Fc modifications can be utilized to drive enhanced biological activity. Although the SDIE mutation used here improved all measured Fc effector functions, other mutations exist that selectively improve NK cell activity, monocyte phagocytosis, neutrophil activation, or complement deposition, offering potentially more precise mechanisms to control infection. Moreover, recent vaccine correlates analysis in nonhuman primates highlighted the complementary activity of monocyte phagocytosis and complement in viral control $(28,51)$. Whether these functions alone may selectively clear the virus and prevent inflammation and pathology remains unclear but could provide critical clues for the strategic engineering of monoclonal antibodies to maximize protection and minimize pathology. 
As the number of COVID-19 cases rise globally, new therapies are urgently needed to treat this highly infectious virus. Here, we characterized the therapeutic functions of the monoclonal antibody CR3022 that binds to a conserved site on the RBD that is not fully blocked in the presence of ACE2, offering therapeutic benefit even after infection has been initiated. The data presented here show no effect of CR3022 as a WT IgG1. Surprisingly, both the Fc-enhanced and Fc-silenced variants of CR3022 showed an antiviral benefit in mice but resulted in dichotomous treatment-associated pathology. Interestingly, the same pathological phenotype was observed in hamsters with the Fc-enhanced variant, highlighting the consistent disease-enhancing phenotype of highly functional non-neutralizing monoclonal variants. With the rapid discovery of novel neutralizing antibodies and pan-cross-reactive $\mathrm{CoV}$ antibodies, coupled to rapid Fc engineering, enabling the potential to deeply profile the involvement of all Fc receptors (including Fc $\gamma R$ 1, Fc $\gamma R 2 a, F_{c} \gamma 2 b, F_{c} \gamma$ R3a, $\mathrm{Fc} \gamma \mathrm{R} 3 \mathrm{~b}, \mathrm{FcRn}$, as well as noncanonical Fc receptors) and the generation of functionally optimized antibodies with appropriate half-lives, the development of therapeutics with the highest clinical benefit is possible.

\section{Methods}

Cell lines. THP-1 cells, originally isolated from a 1-year-old male human (ATCC), were maintained in RPMI supplemented with 10\% fetal bovine serum, L-glutamine, HEPES, penicillin/streptomycin, and 0.01\% $\beta$-mercaptoethanol. Vero E6 cells, from BEI Resources, National Institute of Allergy and Infectious Diseases (NIAID) NIH: VERO C1008 (E6), African green monkey kidney, Working Bank NR-596, were maintained in humidified incubators at $37^{\circ} \mathrm{C}$ and $5 \% \mathrm{CO}_{2}$ in Dulbecco's modified Eagle medium (DMEM) with GlutaMAX and sodium pyruvate supplemented with $10 \%(v / v)$ certified US-origin heat-inactivated fetal bovine serum (HI-FBS).

Viruses. SARS-CoV-2 USA-WA1/2020 (54) was propagated on Vero E6 cells in DMEM supplemented with $2 \%$ HI-FBS, GlutaMAX, sodium pyruvate, nonessential amino acids, and antibiotic-antimycotic. At 62 hours, the supernatant was harvested and clarified by centrifugation. The final concentration of HI-FBS was diluted to $10 \%(v / v)$ prior to cryopreservation at $-80^{\circ} \mathrm{C}$. Final passage was VERO +3 , Vero E6 $+2(10 t$ NSU-V004). The sequence of this stock was identical to the published reference consensus sequence (54).

For in vivo studies, a recombinant SARS-CoV-2 mouse-adapted variant was constructed by introduction of 2 amino acid changes in the ACE2 binding pocket. Virus stocks were grown on Vero cells and titered by plaque assay as previously described by our group (36).

Animals. Female 12-month-old BALB/c mice were obtained from Envigo (strain 047). All animal work was approved by Institutional Animal Care and Use Committee at University of North Carolina at Chapel Hill. Syrian golden hamsters, 6-7 weeks old, were from and maintained at University of Texas Medical Branch.

Plasmid design. To create the CR3022 variants, gene blocks were designed containing the Fc domain of IgG1 and previously defined, individual Fc point-mutant backbones with known differences in binding to Fc receptors and functional differences (32-34). These Fc domains were cloned into individual pUC donor plasmids. In addition, 3 pUC plasmids encoding the variable heavy chain, a furin P2A sequence, or the variable light chain were designed surrounded by BsaI sites. In addition to the 4 pUC plasmids, a destination vector was cloned with an IL-2 secretion signal, the suicide gene ccdB surrounded by BsaI sites, and the kappa light chain sequence. The 4 pUC donor plasmids and the destination vector were combined in a single digestion-ligation reaction, using Golden Gate cloning, to create full IgG molecules with the same CR3022 antigen-binding (Fab) domain but different Fc domains.

Protein expression and purification. The RBD (residues 319-529) of SARS-CoV-2 S protein (GenBank: MN975262.1) were subcloned into a pVRC vector with a C-terminal SBP-tag.

The CR3022 (GenBank: DQ168569 and DQ168570), B38 (27), and 0012C10 and 0012E4 antibodies (provided in-house) were produced in 293F suspension cells grown in FreeStyle 293 Expression media (Gibco, Thermo Fisher Scientific). Cells were transfected with Polyethylenimine (PEI; Polysciences) at $1 \mu \mathrm{g} / \mu \mathrm{L}$ in a ratio of $3 \mu \mathrm{g}$ PEI to $1 \mu \mathrm{g}$ DNA. Supernatants were harvested 5 days posttransfection, and antibody was purified using protein $\mathrm{G}$ magnetic beads (MilliporeSigma). For in vitro analysis, KZ52 (Mayflower Bioscience, 0260-001) was used as a negative control.

ELISA. ELISA plates were coated with $50 \mathrm{ng}$ /well of antigen in PBS overnight at $4^{\circ} \mathrm{C}$ on a shaker at low speed. The next day, plates were washed 5 times with PBS- $0.05 \%$ Tween-20 (PBST) and blocked in 5\% BSA in PBS for 2 hours at room temperature on a shaker at low speed. Plates were washed 5 times with PBST, and 5-fold serially diluted antibody was added and incubated for 2 hours at room temperature on a shaker at low speed. After the incubation, plates were washed with PBST and anti-human IgG1-HRP was 
added for detection. Plates were incubated for 1 hour at room temperature on a shaker at low speed. Plates were washed with PBST. The ELISA was developed with the addition of TMB (Invitrogen, Thermo Fisher Scientific). The reaction was stopped with $1 \mathrm{M} \mathrm{H}_{2} \mathrm{SO}_{4}$. Signal reading was carried out at $450 \mathrm{~nm}$ (reference wavelength of 570). Data were reference value and background corrected.

$A D C P$ assay. The ADCP assay was adapted from Ackerman et al. (55). Briefly, antigen was biotinylated using sulfo-NHS (N-hydroxysulfosuccinimide, Pierce, Thermo Fisher Scientific, A39269) LC-LC biotin, coupled to yellow-green fluorescent Neutravidin $1 \mu \mathrm{m}$ beads (Invitrogen, Thermo Fisher Scientific, F8776) for 2 hours at $37^{\circ} \mathrm{C}$ and washed 3 times in $0.1 \%$ BSA in PBS. The coupled beads were resuspended to a final volume of $10 \mu \mathrm{g} / \mathrm{mL}$. A total of $10 \mu \mathrm{L} /$ well of coupled beads were added to 96-well plates with $100 \mu \mathrm{L} /$ well of antibodies at a concentration of $5 \mu \mathrm{g} / \mathrm{mL}, 1 \mu \mathrm{g} / \mathrm{mL}, 0.2 \mu \mathrm{g} / \mathrm{mL}$, and $0.04 \mu \mathrm{g} / \mathrm{mL}$ for 2 hours at $37^{\circ} \mathrm{C}$ to form immune complexes. After incubation, the immune complexes were spun down and the supernatant was removed. THP-1 cells (ATCC) were added at a concentration of $2.5 \times 10^{4}$ cells/well and incubated for 18 hours at $37^{\circ} \mathrm{C}$. After incubation, the plates were spun down, the supernatant was removed, and cells were fixed with $4 \%$ PFA for 20 minutes. Fluorescence was acquired with an Intellicyt iQue. Phagocytic score was calculated using the following formula: (percentage of $\mathrm{FITC}^{+}$cells $\times$the geometric MFI of the $\mathrm{FITC}^{+}$cells)/10,000.

ADNP assay. The ADNP assay was adapted from Karsten et al. (56). Antigens were coupled to beads and immune complexes were formed as described for ADCP. Neutrophils were isolated from freshly drawn whole blood. Erythrocytes were lysed with ammonium-chloride potassium lysis buffer $\left(150 \mathrm{mM} \mathrm{NH}_{4} \mathrm{Cl}_{1} 10\right.$ $\mathrm{mM} \mathrm{KHCO}, 0.1 \mathrm{mM} \mathrm{Na}_{2}$ EDTA, $\mathrm{pH} 7.4$ ), and leukocytes were separated out by centrifugation, $500 \mathrm{~g}$ for 5 minutes at room temperature. Leukocytes were washed with cold PBS, resuspended in R10, and added to plates at a concentration of $5 \times 10^{4}$ cells/well. The plates were incubated for 1 hour at $37^{\circ} \mathrm{C}$. The neutrophil marker CD66b (Pacific Blue-conjugated anti-CD66b; BioLegend, 305112) was used to stain cells. Cells were fixed for 20 minutes in 4\% paraformaldehyde (PFA). Fluorescence was acquired with an Intellicyt iQue, and the phagocytic score was calculated as described for ADCP.

$A D C D$ assay. The ADCD assay was adapted from Fischinger et al. (57). Antigen was coupled to red fluorescent Neutravidin $1 \mu \mathrm{m}$ beads (Invitrogen, Thermo Fisher Scientific, F8775) as described for ADCP. Immune complexes were formed by incubating $10 \mu \mathrm{L}$ of coupled beads with $50 \mu \mathrm{L}$ of antibody at concentrations of $50 \mu \mathrm{g} / \mathrm{mL}, 10 \mu \mathrm{g} / \mathrm{mL}, 2 \mu \mathrm{g} / \mathrm{mL}$, and $0.4 \mu \mathrm{g} / \mathrm{mL}$ for 2 hours at $37^{\circ} \mathrm{C}$. Plated were spun down, and immune complexes were washed with PBS. Lyophilized guinea pig complement (Cedarlane, CL4051) was resuspended in $1 \mathrm{~mL}$ of cold water, diluted 1:50 in GVB++ (gelatin veronal buffer and additional $\mathrm{Ca}^{+}$and $\mathrm{Mg} 2^{+}$, Boston BioProducts, IBB-300X), and added to the immune complexes. The plates were incubated for 20 minutes at $37^{\circ} \mathrm{C}$, and the reaction was stopped by washing the plates twice with $15 \mathrm{mM}$ EDTA in PBS. To detect complement deposition, plates were incubated with fluorescein-conjugated goat anti-guinea pig complement C3 (MP Biomedicals, 0855385) for 15 minutes in the dark. Fluorescence was acquired with an Intellicyt iQue.

ADNKA (NK activation). Human NK cells were isolated from buffy coats using RosetteSep NK cell enrichment kit (StemCell Technologies) and Ficoll separation. The isolated NK cells were rested overnight at 1.5 $\times 10^{6} \mathrm{cells} / \mathrm{mL}$ in IL- 15 at $37^{\circ} \mathrm{C}$. ELISA plates were coated with antigen at $300 \mathrm{ng} /$ well and incubated for 2 hours at $37^{\circ} \mathrm{C}$. Plates were blocked with $5 \%$ BSA in PBS overnight at $4^{\circ} \mathrm{C}$. The next day, $100 \mu \mathrm{L}$ of antibodies, at a concentration of $5 \mu \mathrm{g} / \mathrm{mL}$, were added to the plates. Plates were incubated for 2 hours at $37^{\circ} \mathrm{C}$ to form immune complexes. After the incubation, NK cells were added to the plates at $5 \times 10^{4}$ cells/well in R10 supplemented with anti-CD107a PE-Cy5, Brefeldin A (MilliporeSigma, B7651-5MG), and GolgiStop (BD Biosciences, 555802). Plates were incubated for 5 hours at $37^{\circ} \mathrm{C}$. Following the incubation, NK cells were stained for the surface markers with anti-CD56 PE-Cy7, anti-CD16 APC-Cy7, and anti-CD3 Pacific Blue (BD Biosciences, 557747, 557758, 558124). NK cells were fixed and permeabilized with Fix\&Perm cell permeabilization kit (Invitrogen, Thermo Fisher Scientific). Cells were incubated with anti-MIP-1 $\beta$ PE and anti-IFN- $\gamma$ FITC (BD Biosciences, 550078, 340449) to stain for intracellular markers. Cells were acquired on an Intellicyt iQue.

FcR binding. A multiplex assay was used to determine FcR binding as described in Brown et al. (58, 59). A 2-step carbodiimide reaction was used to couple antigen to Magplex Luminex beads. Beads were activated for 30 minutes at room temperature using $100 \mathrm{mM}$ monobasic sodium phosphate, $\mathrm{pH}$ 6.2, with $5 \mathrm{mg} / \mathrm{mL}$ sulfo-NHS and $5 \mathrm{mg} / \mathrm{mL}$ ethyl dimethylaminopropyl carbodiimide hydrochloride. Beads were then washed with $50 \mathrm{mM}$ 2-(N-Morpholino)ethanesulfonic acid (MES), pH 5.0, and incubated with 25 $\mu \mathrm{g}$ of antigen in $50 \mathrm{mM} \mathrm{MES}$, pH 5.0, for 2 hours on a rotator. The coupled beads were blocked in Blocking Buffer (PBS, 0.1\% BSA, 0.02\% Tween-20, 0.05\% Azide, pH 7.4). After blocking, coupled beads were washed in PBS-Tween, resuspended in PBS, and stored at $4^{\circ} \mathrm{C}$. 
For the detection of FcR binding, FcRs with an AviTag were biotinylated using a BirA500 kit (Avidity) per the manufacturer's instructions. Coupled beads were diluted to a concentration of 100 microspheres per antigen $/ \mu \mathrm{L}$ in $0.1 \%$ BSA in PBS. Antibodies were serially diluted in $0.1 \%$ BSA in PBS; mixed with diluted beads in a black, clear-bottom, 384-well plate; and incubated at $4^{\circ} \mathrm{C}$ for 16 hours, shaking at $900 \mathrm{rpm}$. After the incubation, plates were washed with $0.1 \%$ BSA in PBS. FcRs were incubated with streptavidin-PE (Prozyme, PJ31S) for 10 minutes. PE-labeled FcRs were added to plates and incubated for 1 hour at room temperature on a shaker. Plates were washed with $0.1 \%$ BSA in PBS and resuspended in Qsol Buffer (Intellicyt). Fluorescence was acquired on the Intellicyt iQue.

In vitro plaque reduction neutralization assay. The day prior to assay, VeroE6 cells were seeded to a density of $8 \times 10^{5}$ cells/well in 6-well plates. SARS-CoV-2 was diluted in DMEM with GlutaMAX and sodium pyruvate supplemented with $1 \times$ antibiotic-antimycotic and $2 \% \mathrm{HI}-\mathrm{FBS}$ to $1000 \mathrm{PFU} / \mathrm{mL}$ (target 100 PFU per well). Antibody was serially diluted in Dulbecco's PBS, and an equal volume of diluted SARS-CoV-2 was added, mixed, and incubated for 1 hour at $37^{\circ} \mathrm{C}$ before plating on 6 -well plates (200 $\mu \mathrm{L}$ in triplicate). Following a 1 -hour incubation at $37^{\circ} \mathrm{C}$ with periodic rocking, they were overlaid with a 1:1 mixture of 2.5\% (w/v) Avicel RC-591 (provided by DuPont Nutrition \& Health) prepared in distilled water and $2 \times$ Temin's Modified Eagle Medium (Thermo Fisher Scientific) supplemented with $10 \%$ HI-FBS, $2 \times$ GlutaMAX, and $2 \times$ antibiotic-antimycotic. Following a 2-day incubation at $37^{\circ} \mathrm{C}$ and $5 \% \mathrm{CO}_{2}$, plates were fixed with $10 \%$ neutral buffered formalin for removal from biocontainment and stained with a solution of $0.2 \%$ Gentian Violet and $10 \%$ neutral buffered formalin. Plates were rinsed under water and plaques were enumerated. Percentage neutralization was calculated from vehicle/ virus-only control wells.

In vivo challenge. Female 12-month-old BALB/c mice were treated prophylactically (12 hours before infection) or therapeutically (12 hours after infection) with $200 \mu \mathrm{g}$ or $100 \mu \mathrm{g}$ of antibody through the intraperitoneal route. Each group contained 5 mice. Mice were challenged i.n. with $10^{5}$ PFU of mouse-adapted SARS-CoV-2, representing $0.69 \times 10^{5} \mathrm{TCID}_{50}$, which falls clearly in the range of viral loads observed in hospitalized patients (60). Weight was monitored on days 0,1 , and 2 after infection. Mice were sacrificed 2 days after infection, and lung viral titer was determined by plaque assay. Although the Fc variants experience different half-lives in vivo, the studies performed here were short; but half-life should be considered for longer studies.

Adult hamsters were microchipped a day prior to experimental challenge. On day 0 , hamsters were anesthetized with ketamine/xylazine and challenged with SARS-CoV-2 by the i.n. route using a $10^{7}$ $\mathrm{TCID}_{50}$ (or $10^{6} \mathrm{PFU} / \mathrm{mL}$ ) dose in a total volume of $100 \mu \mathrm{L}$. The final challenge dose was $10^{4} \mathrm{PFU}$ diluted in sterile PBS. Body weight and body temperature were measured each day, starting at day 0 . On day 1 postchallenge $(1 \mathrm{dpc}$ ) hamsters were treated with $5 \mathrm{mg} / \mathrm{kg}$ of monoclonal antibodies diluted in $0.5 \mathrm{~mL}$ of sterile PBS via the intraperitoneal (i.p.) route. The control group got an equal volume of sterile PBS via the same i.p. route. On $3 \mathrm{dpc}$ all animals were sacrificed. At necropsy, lungs were harvested for all groups. Left lungs were fixed with 10 volumes of fresh $10 \%$ formalin; right lungs were frozen in $5 \mathrm{~mL}$ lysogeny broth from Thermo Fisher Scientific for viral load analysis. Tissue sections were homogenized in bead beater tubes (Thomas Scientific) and weighed, and supernatants were titrated per standard protocol. Briefly, $100 \mu \mathrm{L}$ of a $10 \times$ dilution of supernatants was incubated in 96 -well plates for 1 hour, and supernatants were replaced by methyl cellulose overlay and incubated for 3 days at $5 \% \mathrm{CO}_{2}$ and $37^{\circ} \mathrm{C}$. Plates were fixed with formalin and removed from the biosafety level 4 facility, after which the plates were inactivated and immunostained, and the plaques were counted to obtain viral titers.

Statistics. All data were visualized and analyzed in GraphPad Prism. Nonparametric tests were performed as described in figure legends. Where applicable, significance was determined as $*<0.05$, ${ }^{* *} P<0.01, * * * P<0.001$.

Study approval. Primary human innate immune cells were isolated from fresh peripheral blood samples collected by the Massachusetts General Hospital (MGH) blood bank. All subjects provided informed consent, and the study was approved by the MGH Institutional Review Board. All subjects were older than 18 years of age, and samples were deidentified prior to use.

All mouse work was approved by the Institutional Animal Care and Use Committee at the University of North Carolina at Chapel Hill. The animal protocols for the hamster models were approved by the Institutional Animal Care and Use Committee of the University of Texas Medical Branch. 


\section{Author contributions}

CA, MDS, AG, TS, BDJ, JG, DRW, AB, RB, and GA designed the study. CA, SF, MDS, JB, AS, SRL, NAK, CM, AH, RJ, NS, PT, TZ, JL, AZ, and CL performed experiments. MB, DRW, and JRD provided reagents. CA performed all analysis.

\section{Acknowledgments}

We thank Nancy Zimmerman, Bruce Walker, Mark and Lisa Schwartz, and Terry and Susan Ragon for their support. We would also like to thank Bing Chen, Kizzmekia Corbett, Aaron Schmidt, Jared Feldman, Blake Hauser, and Tim Caradonna for protein production efforts and Sierra Downs for technical support. The following reagent was obtained through BEI Resources, NIAID, NIH: VERO C1008 (E6), Kidney (African green monkey), Working Cell Bank, NR-596. The SARS-CoV-2 starting material was provided by the World Reference Center for Emerging Viruses and Arboviruses, with Natalie Thornburg (nax3@cdc.gov) as the CDC principal investigator. Avicel RC-591 was provided by DuPont Nutrition \& Health. We would like to thank Deborah Gakpo, Jillian Bensko, Sudeshna Fisch, Meghan Travers, Shaghayaegh Habibi, Yuezhou Chen, Adam Zuiani, and Felipe N. Lelis for organizing and collecting human samples used for the cloning of monoclonal antibodies. We would also like to thank Massachusetts Consortium on Pathogen Readiness, the Samana Cay MGH Scholar program, and an anonymous donor for financial support. This work was supported by the NIH (3R37AI080289-11S1) and the NIAID, NIH (1U01CA260476-01, U19 AI135995, NIH AI121394, AI139538).

Address correspondence to: Galit Alter, 400 Technology Square, Cambridge, Massachusetts 02139, USA. Phone: 857.268.7003; Email: galter@mgh.harvard.edu.

1. WHO. Weekly Epidemiological Update on COVID-19. https://www.who.int/publications/m/item/weekly-epidemiological-update-8-december-2020. Updated December 8, 2020. Accessed December 14, 2020.

2. Peiris JS. Severe acute respiratory syndrome (SARS). J Clin Virol. 2003;28(3):245-7.

3. de Wit E, et al. SARS and MERS: recent insights into emerging coronaviruses. Nat Rev Microbiol. 2016;14(8):523-34.

4. Ruan S. Likelihood of survival of coronavirus disease 2019. Lancet Infect Dis. 2020; 20(6):630-631.

5. Devaux CA, et al. New insights on the antiviral effects of chloroquine against coronavirus: what to expect for COVID-19? Int $J$ Antimicrob Agents. 2020;55(5):105938.

6. Grein J, et al. Compassionate use of remdesivir for patients with severe Covid-19. N Engl J Med. 2020;382(24):2327-2336

7. Song Z, et al. From SARS to MERS, thrusting coronaviruses into the spotlight. Viruses. 2019;11(1):E59.

8. Jin Y, et al. Human monoclonal antibodies as candidate therapeutics against emerging viruses. Front Med. 2017;11(4):462-470

9. Widjaja I, et al. Towards a solution to MERS: protective human monoclonal antibodies targeting different domains and functions of the MERS-coronavirus spike glycoprotein. Emerg Microbes Infect. 2019;8(1):516-530.

10. Corti D, et al. Prophylactic and postexposure efficacy of a potent human monoclonal antibody against MERS coronavirus. Proc Natl Acad Sci U S A. 2015;112(33):10473-10478.

11. $\mathrm{Li} \mathrm{Y}$, et al. A humanized neutralizing antibody against MERS-CoV targeting the receptor-binding domain of the spike protein. Cell Res. 2015;25(11):1237-1249.

12. Du L, et al. The spike protein of SARS-CoV--a target for vaccine and therapeutic development. Nat Rev Microbiol. 2009;7(3):226-236.

13. Zhao J, et al. Passive immunotherapy with dromedary immune serum in an experimental animal model for Middle East respiratory syndrome coronavirus infection. J Virol. 2015;89(11):6117-6120.

14. Li Q, et al. Early transmission dynamics in Wuhan, China, of Novel coronavirus-infected pneumonia. $N$ Engl J Med. 2020;382(13):1199-1207.

15. Zhang S, et al. Estimation of the reproductive number of novel coronavirus (COVID-19) and the probable outbreak size on the Diamond Princess cruise ship: a data-driven analysis. Int J Infect Dis. 2020;93:201-204.

16. Cheng ZJ, Shan J. 2019 Novel coronavirus: where we are and what we know. Infection. 2020;48(2):155-163.

17. Maimuna M, Mandi KD. Early transmissibility assessment of a novel coronavirus in Wuhan, China [preprint]. https:// doi:10.2139/ssrn.3524675. Posted on SSRN January 24, 2020.

18. Sanche S, et al. High contagiousness and rapid spread of severe acute respiratory syndrome coronavirus 2. Emerg Infect Dis. 2020;26(7):1470-1477.

19. Liu L, et al. Anti-spike IgG causes severe acute lung injury by skewing macrophage responses during acute SARS-CoV infection. JCI Insight. 2019;4(4):e123158.

20. Tian X, et al. Potent binding of 2019 novel coronavirus spike protein by a SARS coronavirus-specific human monoclonal antibody. Emerg Microbes Infect. 2020;9(1):382-385.

21. Yuan M, et al. A highly conserved cryptic epitope in the receptor-binding domains of SARS-CoV-2 and SARS-CoV [preprint] https://doi:10.1101/2020.03.15.992883. Posted on bioRxiv March 17, 2020.

22. ter Meulen J, et al. Human monoclonal antibody combination against SARS coronavirus: synergy and coverage of escape mutants. PLoS Med. 2006;3(7):e237.

23. Hamming I, et al. Tissue distribution of ACE2 protein, the functional receptor for SARS coronavirus. A first step in understand- 
ing SARS pathogenesis. J Pathol. 2004;203(2):631-637.

24. Gunn BM, et al. A role for Fc function in therapeutic monoclonal antibody-mediated protection against Ebola virus. Cell Host Microbe. 2018;24(2):221-233.e5.

25. Saphire EO, et al. Systematic analysis of monoclonal antibodies against Ebola virus GP defines features that contribute to protection. Cell. 2018;174(4):938-952.e13.

26. Ziegler CGK, et al. SARS-CoV-2 receptor ACE2 is an interferon-stimulated gene in human airway epithelial cells and is detected in specific cell subsets across tissues. Cell. 2020;181(5):1016-1035.e19.

27. Wu Y, et al. A noncompeting pair of human neutralizing antibodies block COVID-19 virus binding to its receptor ACE2. Science. 2020;368(6496):1274-1278.

28. Yu J, et al. Bibliometric analysis of Ebola research indexed in web of science and scopus (2010-2020). Biomed Res Int. 2020;2020:5476567.

29. Takada A, Kawaoka Y. Antibody-dependent enhancement of viral infection: molecular mechanisms and in vivo implications. Rev Med Virol. 2003;13(6):387-398

30. Taylor A, et al. Fc receptors in antibody-dependent enhancement of viral infections. Immunol Rev. 2015;268(1):340-364.

31. Overdijk MB, et al. Crosstalk between human IgG isotypes and murine effector cells. J Immunol. 2012;189(7):3430-3438.

32. Moore GL, et al. Engineered Fc variant antibodies with enhanced ability to recruit complement and mediate effector functions. MAbs. 2010;2(2):181-189.

33. Lazar GA, et al. Engineered antibody Fc variants with enhanced effector function. Proc Natl Acad Sci U S A. 2006;103(11):4005-4010.

34. Leabman MK, et al. Effects of altered Fc $\gamma \mathrm{R}$ binding on antibody pharmacokinetics in cynomolgus monkeys. MAbs. 2013;5(6):896-903.

35. Nimmerjahn F, et al. FcgammaRIV: a novel FcR with distinct IgG subclass specificity. Immunity. 2005;23(1):41-51.

36. Dinnon KH, et al. A mouse-adapted SARS-CoV-2 model for the evaluation of COVID-19 medical countermeasures [preprint] https://doi:10.1101/2020.05.06.081497. Published on bioRxiv May 7, 2020.

37. Sia SF, et al. Pathogenesis and transmission of SARS-CoV-2 in golden hamsters. Nature. 2020;583(7818):834-838.

38. Imai M, et al. Syrian hamsters as a small animal model for SARS-CoV-2 infection and countermeasure development. Proc Natl Acad Sci U S A. 2020;117(28):16587-16595.

39. Wang SF, et al. Antibody-dependent SARS coronavirus infection is mediated by antibodies against spike proteins. Biochem Biophys Res Commun. 2014;451(2):208-214.

40. Yang ZY, et al. Evasion of antibody neutralization in emerging severe acute respiratory syndrome coronaviruses. Proc Natl Acad Sci U S A. 2005;102(3):797-801.

41. Yip MS, et al. Antibody-dependent infection of human macrophages by severe acute respiratory syndrome coronavirus. Virol $J$. 2014;11:82.

42. Jaume M, et al. Anti-severe acute respiratory syndrome coronavirus spike antibodies trigger infection of human immune cells via a pH- and cysteine protease-independent Fc $\gamma \mathrm{R}$ pathway. J Virol. 2011;85(20):10582-10597.

43. Katzelnick LC, et al. Antibody-dependent enhancement of severe dengue disease in humans. Science. 2017;358(6365):929-932

44. Ayala-Nunez NV, et al. How antibodies alter the cell entry pathway of dengue virus particles in macrophages. Sci Rep. 2016;6:28768.

45. Puelles VG, et al. Multiorgan and renal tropism of SARS-CoV-2. N Engl J Med. 2020;383(6):590-592.

46. Sungnak W, et al. SARS-CoV-2 entry factors are highly expressed in nasal epithelial cells together with innate immune genes. Nat Med. 2020;26(5):681-687.

47. Latvala S, et al. Distribution of FcRn across species and tissues. J Histochem Cytochem. 2017;65(6):321-333.

48. Kuo TT, et al. Neonatal Fc receptor: from immunity to therapeutics. J Clin Immunol. 2010;30(6):777-789.

49. Mahan AE, et al. Antigen-specific antibody glycosylation is regulated via vaccination. PLoS Pathog. 2016;12(3):e1005456.

50. Chung AW, et al. Dissecting polyclonal vaccine-induced humoral immunity against HIV using systems serology. Cell. 2015;163(4):988-998.

51. Mercado NB, et al. Single-shot Ad26 vaccine protects against SARS-CoV-2 in rhesus macaques. Nature. 2020;586(7830):583-588.

52. Eli Lilly. Lilly's Neutralizing Antibody Bamlanivimab (LY-CoV555) Receives FDA Emergency Use Authorization for the Treatment of Recently Diagnosed COVID-19. https://investor.lilly.com/news-releases/news-release-details/lillys-neutralizing-antibodybamlanivimab-ly-cov555-receives-fda. Updated November 9, 2020. Accessed November 12, 2020.

53. Barnhart BC, Quigley M. Role of Fc-Fc $\gamma \mathrm{R}$ interactions in the antitumor activity of therapeutic antibodies. Immunol Cell Biol. 2017;95(4):340-346

54. Harcourt J, et al. Severe acute respiratory syndrome coronavirus 2 from patient with coronavirus disease, United States. Emerg Infect Dis. 2020;26(6):1266-1273.

55. Ackerman ME, et al. A robust, high-throughput assay to determine the phagocytic activity of clinical antibody samples. $J$ Immunol Methods. 2011;366(1-2):8-19.

56. Karsten CB, et al. A versatile high-throughput assay to characterize antibody-mediated neutrophil phagocytosis. J Immunol Methods. 2019;471:46-56.

57. Fischinger S, et al. A high-throughput, bead-based, antigen-specific assay to assess the ability of antibodies to induce complement activation. J Immunol Methods. 2019;473:112630.

58. Brown EP, et al. Multiplexed Fc array for evaluation of antigen-specific antibody effector profiles. J Immunol Methods. 2017;443:33-44.

59. Brown EP, et al. High-throughput, multiplexed IgG subclassing of antigen-specific antibodies from clinical samples. $J$ Immunol Methods. 2012;386(1-2):117-123.

60. Wolfel R, et al. Virological assessment of hospitalized patients with COVID-2019. Nature. 2020;581(7809):465-469. 